Original Research Paper

\title{
Uniform Twister Plane Generator
}

\author{
${ }^{1}$ Aleksei F. Deon and ${ }^{2}$ Yulian A. Menyaev \\ ${ }^{I}$ Department of Information Systems and Computer Science, \\ N.E. Bauman Moscow State Technical University, Moscow, Russia \\ ${ }^{2}$ Winthrop P. Rockefeller Cancer Institute, University of Arkansas for Medical Sciences, Little Rock, AR, USA
}

Article history

Received: 01-11-2017

Revised: 07-02-2018

Accepted: 22-02-2018

Corresponding Author:

Yulian A. Menyaev

Winthrop P. Rockefeller Cancer

Institute, University of

Arkansas for Medical Sciences,

Little Rock, AR, USA

Email: yamenyaev@uams.edu

\begin{abstract}
Random plane generators may use various types of the random number algorithms to create multidimensional planes. At the same time, the discrete Descartes random planes have to be uniform. The matter is that using the concept of the uncontrolled random generation may lead to a result of weak quality due to initial sequences having either insufficient uniformity or skipping of the random numbers. This article offers a new approach for creating the absolute twisting uniform two-dimensional Descartes planes based on a model of complete twisting sequences of uniform random variables without repetitions or skipping. The simulation analyses confirm that the resulted random planes have an absolute uniformity. Moreover, combining the parameters of the original complete uniform sequences allows a significant increase in the number of created planes without using additional random access memory.
\end{abstract}

Keywords: Pseudorandom Number Generator, Stochastic Sequences, Congruential Numbers, Twister Generator, Random Plane, Random Field

\section{Introduction}

In our previous studies (Deon and Menyaev, 2016a; 2016 ; 2017) there were proposed several pseudorandom number generators, particularly nsDeonYuliTwist32D, which offers a technique of using no congruential twisting array. This generator allows the creation of absolutely complete twister uniform sequences having various lengths.

The direction of Random Plane (RP) Generators (RPG) employs a stochastic process at the time of creating the points distributed on $N$-dimensional plane. Here we consider a two-Dimensional (2D) plane only. Other discrete-dimensional planes have the same initial properties. Each coordinate of RP-generated points may belong to its own Random Field (RF). An analysis of the last sources sums up the following selected types of random fields: Conditional RF (Quattoni et al., 2004; Sutton and McCallum, 2012), Markov RF (Sarawagi and Cohen, 2004; Bekkerman et al., 2006), Gaussian RF (Rimstad and Omre, 2014), uniformed RF (Xiao, 2010) and others (Qi et al., 2004; Dachian and Nahapetian, 2009). In the application areas the RPGs are often applied in graphical images (Kumar and Hebert, 2003), phone systems (Sung and Jurafsky, 2009), advertising applications, etc. Next, the RPGs are actively used in fundamental studies, starting from 2D theoretical modeling (Gnedenko, 1998; Feller, 2008), Monte Carlo plane simulation (Newman and Barkema, 1996; Spanos and
Zeldin, 1998), factorial development (Kim and Zabih, 2002), realizations for training systems (Sha and Pereira, 2003 ), etc. and biomedical engineering (Menyaev and Zharov, 2005; 2006a; 2006b; Menyaev et al., 2013; 2016; Koonce et al., 2017).

The principles of all these studies are based on the conception of random planes, in which the Descartes plane features have to satisfy the following properties: (1) The generation process has to provide the uniqueness (i.e., no repetitions) of each point on the plane and (2) the generation process has to keep the completeness (i.e., without skipping) for all created points. These properties should be considered as a 'natural filter' for choosing the random number generator.

Let's consider two of them in brief. If the generation uses the twister generator MT19937 (Matsumoto and Nishimura, 1998; Matsumoto et al., 2006; 2007; Saito and Matsumoto, 2008), then the result of this attempt is very discussable since this generator in DieHard Tests (Berger and Zorn, 2006; Novark and Berger, 2010; Alani, 2010) demonstrates a uniqueness level of 0.7 , which is equivalent to the level of repeatability $1-0.7=0.3$. On the other hand, we may use the twister generator nsDeonYuliTwist32D (Deon and Menyaev, 2017), which is guaranteed to create the complete uniform twisting sequences of an arbitrary size having no repetitions and skipping of elements. Now the question here is: Would it be possible to observe the Descartes properties in the current particular task? 
Let's consider this issue more in detail. For this, let us use the aforementioned twister generator, which is capable of creating complete uniform twisting sequences of arbitrary size. Below is the program code for modeling a grid of the discrete Descartes plane $U \times V$. The program algorithm generates the integer random numbers independently along the $U$ and $V$ independent axes. Matrix $A$ is the discrete plane indicator; its cells $a[u, v] \in A$ with indices $u$ and $v$ correspond to the coordinates of discrete points $\langle u, v>\in U \times V$. The value of cell $a[u, v] \in A$ indicates the quantity of attempts to create independently the corresponding point $\langle u, v\rangle$ on the generated plane. Without loss of generality and in order to visualize the result, we assign the amount of discrete coordinates by given sets $U=V=\{0,1,2,3,4,5$, $6,7\}$, which in the binary representation corresponds to the length $w=3$ bits for each coordinate. According to the previous studies (Deon and Menyaev, 2016b; 2017), let's choose a twister generator nsDeonYuliTwist $32 D$, which operates on the basis of congruential model $x_{i+1}=\left(a x_{i}+c\right)$ $\&$ mask $W$ with constants $a=5, c=1$. The initial values of the twisting sequences are taken here as $x_{0}=1 \in U$ and $x_{0}$ $=4 \in V$. Any other choice of parameters for generation is possible; the essence of the obtained results will not be changed. Program names P040101 and cP040101 are taken by chance. The chosen programming language is $\mathrm{C \#}$ available in Microsoft Visual Studio. The use of other dialects of the older $\mathrm{C}$ versions (i.e., Win32) or $\mathrm{C}++$ (CLR) provides the same results.

using nsDeonYuliTwist32D; // twister uniform generator namespace $\mathrm{P} 040101$

\{ class cP040101

\{ static void Main(string[] args)

\{ uint $\mathrm{w}=3$; cDeonYuliTwist32D GU =

// number bit length new cDeonYuliTwist32D();

GU.x $0=1 ; \quad$ // U sequence beginning

GU.w $=\mathrm{w} ; \quad$ // number bit length

GU.Start(); // GU generator starts

cDeonYuliTwist32D GV = new cDeonYuliTwist32D();

GV.x0 $=4 ; \quad$ // V sequence beginning

GV.w $=\mathrm{w}$; $\quad$ // number bit length

GV.Start();

// GV generator starts

int $\mathrm{N}=1<<$ (int)w; // $\mathrm{U}$ and $\mathrm{V}$ sequences length Console. WriteLine("w $=\{0\} \mathrm{N}=\{1\}$ ", w, N); uint[] $\mathrm{U}=$ new uint[N]; $\quad$ // $\mathrm{U}$ sequence

uint[] $\mathrm{V}=$ new uint[N];

int[,] $\mathrm{A}=$ new int[N,N];

// V sequence

// result matrix

for (int $\mathrm{i}=0 ; \mathrm{i}<\mathrm{N} ; \mathrm{i}++$ ) for (int $\mathrm{j}=0 ; \mathrm{j}<\mathrm{N} ; \mathrm{j}++$ ) $A[\mathrm{i}, \mathrm{j}]=0$;

for (int $\mathrm{i}=0 ; \mathrm{i}<\mathrm{N} ; \mathrm{i}++$ ) // one of the axis

\{ Console.Write $(" \mathrm{i}=\{0,3\} \mid$ ", i);

for (int $\mathrm{j}=0 ; \mathrm{j}<\mathrm{N} ; \mathrm{j}++$ ) // another axis

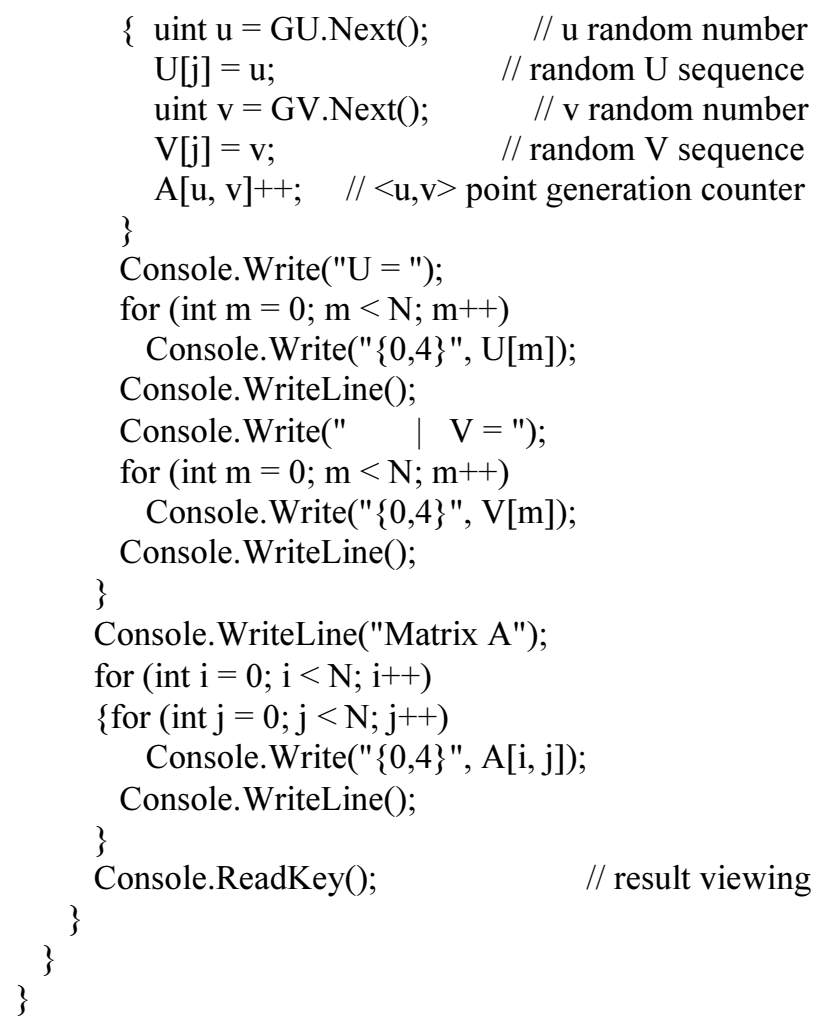

After this code execution the listing below appears:

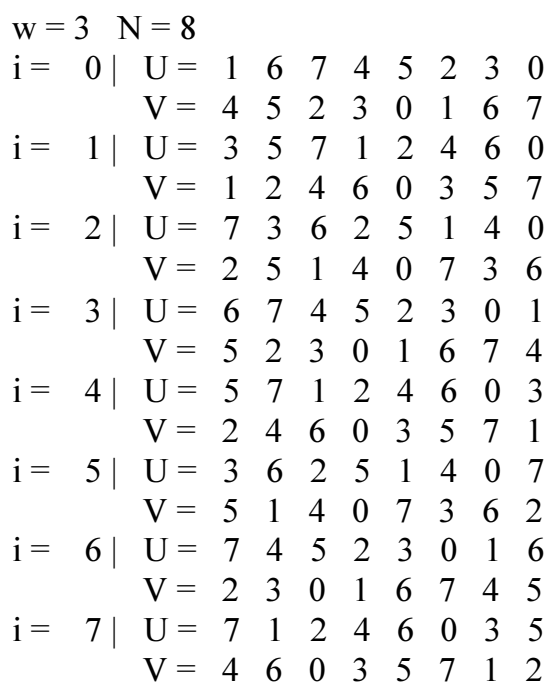

Matrix A

$\begin{array}{llllllll}0 & 0 & 0 & 0 & 0 & 0 & 2 & 6\end{array}$

$\begin{array}{llllllll}0 & 0 & 0 & 0 & 3 & 0 & 3 & 2\end{array}$

$\begin{array}{llllllll}3 & 3 & 0 & 0 & 2 & 0 & 0 & 0\end{array}$

$\begin{array}{llllllll}0 & 3 & 0 & 0 & 0 & 2 & 3 & 0\end{array}$

$\begin{array}{llllllll}0 & 0 & 0 & 8 & 0 & 0 & 0 & 0\end{array}$

$\begin{array}{llllllll}5 & 0 & 3 & 0 & 0 & 0 & 0 & 0\end{array}$

$\begin{array}{llllllll}0 & 2 & 0 & 0 & 0 & 6 & 0 & 0\end{array}$

$\begin{array}{llllllll}0 & 0 & 5 & 0 & 3 & 0 & 0 & 0\end{array}$ 
In matrix $A$ the cells with values that differed from 1 , show that the independent generation of coordinates of the points on the plane doesn't ensure uniform distribution of random points $\langle u, v\rangle$. Some points are missed (values 0 ) and others are present several times (values $>1$ ).

So, the aim of this article is to find a solution for the generation of uniform discrete twisting planes, which possess the Descartes property of a single presence of the random points in nodes of the discretization grid.

\section{Theory}

One of the options to represent the discrete Descartes plane is an enumeration of all points in the grid nodes, formed from the values of discretization on the corresponding axes. If the location of axes is independent, the grid has a rectangular view. Moreover, if discretization for both axes is the same and uniform, the grid has a square view.

Let's assume that the square grid includes $N$ points of discretization along each axis. Thus, the total number of grid points is $N \times N=N^{2}$. To set these points in a random way, an algorithm is required that can provide a random move from one point to another.

Now would be a good time to point out and emphasize the following: The plane is random only if moving from one point to another, while creating the plane, utilizes the stochastic process.

In this case, the requirement of Descartes axes, which prescribes a unique representation of each point, has to be kept. The unambiguity is determined by the discretization of the axes. Uniqueness is provided by an appropriate procedure, which does not allow entering each point of the grid twice or more times. The skipping of vertices of the grid isn't allowed either. In other words, each point is presented once during generation of all the grid points. In this case, the total enumeration of the points is $N^{2}$. Following this way, such a grid on the Descartes plane is called uniform and a random Descartes plane, which contains uniform grid, is called uniform Descartes RP.

There are many ways to specify the points on the grid. Let's name a few of them:

- Rectangular left or right filling of the grid, when one of the axes is selected and at each location of discretization of this axis, the points along the discrete points of the other axis are placed on the grid

- Rectangular top or bottom filling of the grid under the same conditions

- Diagonal filling of the grid under the same conditions

- The secondary indexing of the discrete points along the Descartes axes
This is not a whole list of possible techniques. The options to choose aren't limited and can be organized by the designer in any possible way. Note that items (1) (3) create ordinary Descartes planes and item (4) allows obtaining the random Descartes planes, if the secondary index is a result of the stochastic process.

In this article, an option of secondary indexing of the discrete marks on the Descartes axes is adopted. Let's demonstrate this by an example, in which the congruential generation of random numbers $x_{i+1}=\left(a x_{i}+\right.$ c)\&maskW is used as secondary indexing base. In order to visualize the results, we take the complete uniform sequences of random numbers $x \in\{0,1,2,3\}=\left\{00_{2}\right.$, $\left.01_{2}, 10_{2}, 11_{2}\right\}$ having length $w=2$ bits. In this case, each complete sequence contains $N=2^{w}=2^{2}=4$ elements. Without loss of generality, let's assume that $a=1 \in[\overline{1, N-1}]$ and $c=3 \in[\overline{1, N-1}]$. In total, four congruential sequences are possible: $<0,3,2,1>,<1,0$, $3,2>,<2,1,0,3>,<3,2,1,0>$. These sequences allow creating various random tracks on uniform Descartes RP.

If random value $x_{0}=1$ is chosen as an initial value, then the designated generator $G U$ creates the sequence $U$ $=<1,0,3,2>$. From this it follows that the initial random vertex will be located on the vertical part of the grid with horizontal discrete mark 1 along the $U$ axis (Fig. 1).

If the second independent generator, which is designated as $G V$, uses the initial random value $x_{0}=3$, then sequence $V=<3,2,1,0>$ is created. From this it's obvious that the second coordinate has the value of 1 for the initial random point $\langle 1,3\rangle$. The next vertex has coordinates $\langle 0,2\rangle$. Both obtained vertices are connected by an arc, forming the beginning of the random track. Then, vertex $<3,1>$ will be placed on this track. Finally, the vertex with coordinates $<2,0>$ completes the random track. For clarity, the visual representation of this track is shown in Fig. 1.

Regarding the random sequence of secondary indices $<1,0,3,2>$ along the $U$ axis, four sequences along the $V$ axis are possible:

1) $\mathrm{U}=<1,0,3,2>$

$\mathrm{V}=<3,2,1,0>$

2) $\mathrm{U}=<1,0,3,2>$

$\mathrm{V}=<2,1,0,3>$

3) $U=<1,0,3,2>$

$\mathrm{V}=<1,0,3,2>$

4) $\mathrm{U}=<1,0,3,2>$

$\mathrm{V}=<0,3,2,1>$

These sequences $V$ can be interpreted as the left circular shift (Deon and Menyaev, 2016b) of the original sequence $<3,2,1,0>$. Figure 2 shows four tracks of an interaction of the pairs of sequences on the $U$ and $V$ axes. These tracks provide an exact one-time generation of each vertex on a grid of the Descartes RP. 


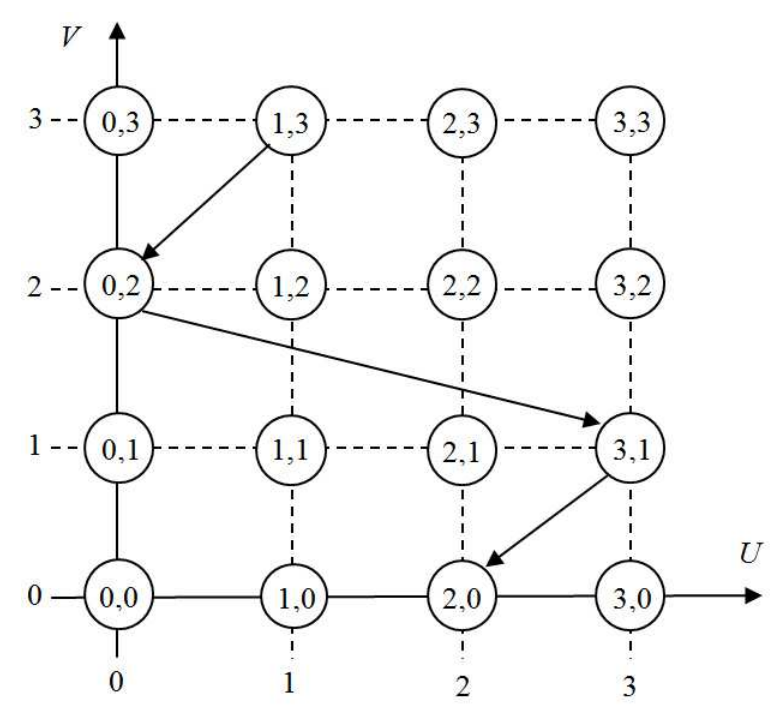

Fig. 1: The initial random track

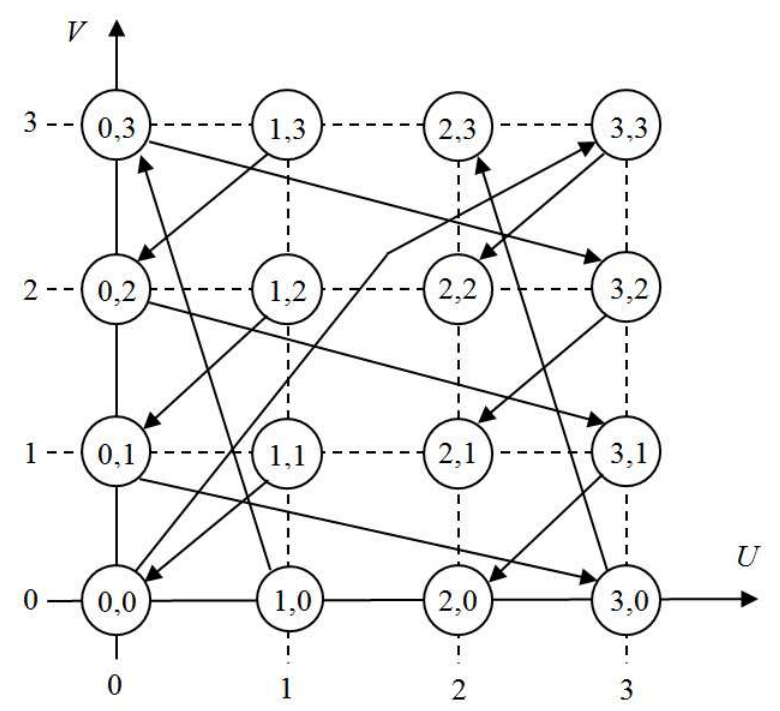

Fig. 2: All the tracks of the random sequences $U$ and $V$

The program code for this task is presented below, in which all the vertices are generated on a grid of the Descartes RP. Random numbers have the length of $w=3$ bits. In each sequences $U$ and $V$ there are $N=2^{w}=2^{3}=8$ random numbers with $x \in[\overline{0, N-1}]=\{0,1,2,3,4,5,6,7\}$. Both sequences $U$ and $V$ are given by a congruential model $x_{i+1}=\left(a x_{i}+c\right) \&$ maskW with coefficients $a=5$ and $c=1$. The first sequence begins with value $x_{0}=1$ and has the form $U=<1,6,7,4,5,2,3,0>$. The second sequence begins with value $x_{0}=4$ and has the form $V=$ $<4,5,2,3,0,1,6,7>$. Each cell of matrix $A$ corresponds to one vertex on a grid of RP. The value of cell $a \in A$ shows how many times the corresponding vertex is generated. Program names P040201 and cP040201 are selected by chance.

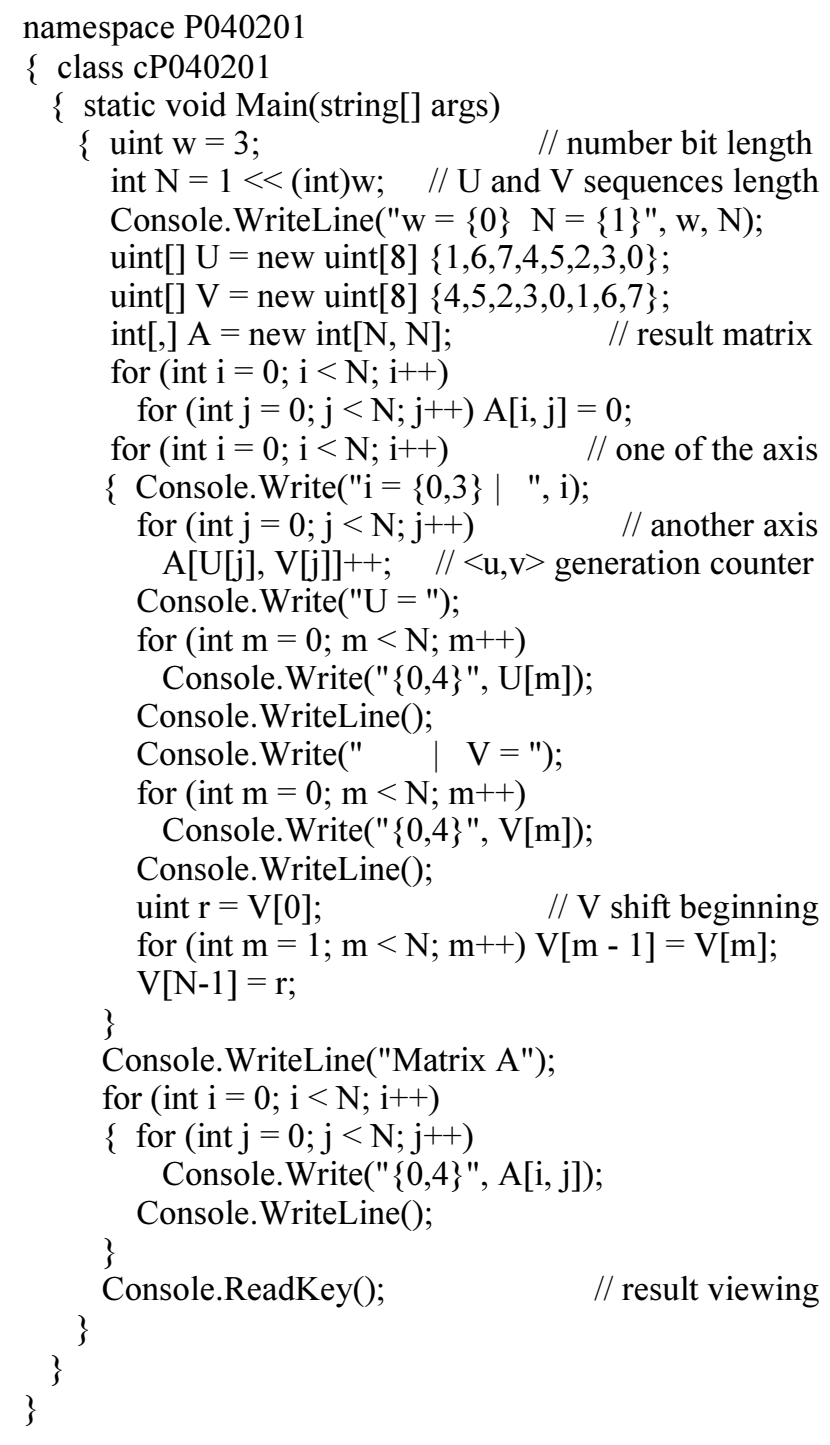

After this code execution the listing below appears:

$$
\begin{aligned}
& \mathrm{W}=3 \quad \mathrm{~N}=8 \\
& \mathrm{i}=0 \mid \mathrm{U}=\begin{array}{llllllll}
1 & 6 & 7 & 4 & 5 & 2 & 3 & 0
\end{array} \\
& \mathrm{~V}=\begin{array}{llllllll}
4 & 5 & 2 & 3 & 0 & 1 & 6 & 7
\end{array} \\
& \mathrm{i}=1 \mid \mathrm{U}=\begin{array}{llllllll}
1 & 6 & 7 & 4 & 5 & 2 & 3 & 0
\end{array} \\
& \mathrm{~V}=\begin{array}{lllllllll}
5 & 2 & 3 & 0 & 1 & 6 & 7 & 4
\end{array} \\
& \mathrm{i}=2 \mid \mathrm{U}=\begin{array}{llllllll}
1 & 6 & 7 & 4 & 5 & 2 & 3 & 0
\end{array} \\
& \mathrm{~V}=\begin{array}{llllllll}
2 & 3 & 0 & 1 & 6 & 7 & 4 & 5
\end{array} \\
& \mathrm{i}=3 \mid \mathrm{U}=1 \begin{array}{llllllll}
1 & 6 & 7 & 4 & 5 & 2 & 3 & 0
\end{array} \\
& \mathrm{~V}=\begin{array}{llllllll}
3 & 0 & 1 & 6 & 7 & 4 & 5 & 2
\end{array} \\
& \mathrm{i}=4 \mid \mathrm{U}=1 \begin{array}{llllllll}
1 & 6 & 7 & 4 & 5 & 2 & 3 & 0
\end{array} \\
& \mathrm{~V}=\begin{array}{lllllllll}
0 & 1 & 6 & 7 & 4 & 5 & 2 & 3
\end{array} \\
& \mathrm{i}=5 \mid \mathrm{U}=1 \begin{array}{llllllll}
1 & 6 & 7 & 4 & 5 & 2 & 3 & 0
\end{array} \\
& \mathrm{~V}=\begin{array}{llllllll}
1 & 6 & 7 & 4 & 5 & 2 & 3 & 0
\end{array} \\
& \mathrm{i}=6 \mid \mathrm{U}=1 \begin{array}{llllllll}
1 & 6 & 7 & 4 & 5 & 2 & 3 & 0
\end{array} \\
& \mathrm{~V}=6 \begin{array}{llllllll}
6 & 7 & 4 & 5 & 2 & 3 & 0 & 1
\end{array} \\
& \mathrm{i}=7 \mid \mathrm{U}=\begin{array}{llllllll}
1 & 6 & 7 & 4 & 5 & 2 & 3 & 0
\end{array} \\
& \mathrm{~V}=\begin{array}{llllllll}
7 & 4 & 5 & 2 & 3 & 0 & 1 & 6
\end{array}
\end{aligned}
$$




\begin{tabular}{cccccccc}
\multicolumn{1}{c}{ Matrix } & $A$ & & & & & \\
1 & 1 & 1 & 1 & 1 & 1 & 1 & 1 \\
1 & 1 & 1 & 1 & 1 & 1 & 1 & 1 \\
1 & 1 & 1 & 1 & 1 & 1 & 1 & 1 \\
1 & 1 & 1 & 1 & 1 & 1 & 1 & 1 \\
1 & 1 & 1 & 1 & 1 & 1 & 1 & 1 \\
1 & 1 & 1 & 1 & 1 & 1 & 1 & 1 \\
1 & 1 & 1 & 1 & 1 & 1 & 1 & 1 \\
1 & 1 & 1 & 1 & 1 & 1 & 1 & 1
\end{tabular}

The values of 1 in the cells of matrix $A$ show that each cell was updated once. This result reflects the single initialization of the corresponding vertices on a grid of uniform Descartes RP.

Similar tests of creating uniform planes with an arbitrary bit length $w$ of the random numbers confirm a uniformity of the received RPs. Replacement of the congruential sequences by the different corresponding twisting sequences shows a similar result when generating the twisting RPs. Thus, the complete uniform twisting sequences can ensure a creation of the complete uniform twisting RPs. Now we may proceed to the generator designing.

\section{Construction and Results}

Program P040201, described in the previous section, utilizes two arrays $U$ and $V$ as initial congruential sequences. Such arrays can be created using the twister generator nsDeonYuliTwist28DA (Deon and Menyaev, 2016b). However, this solution will not be perfect for the case of large planes because it requires a lot of available Random Access Memory (RAM). For many reasons it may be unavailable on a computer. This limitation can be overcome by using the twister generator nsDeonYuliTwist32D (Deon and Menyaev, 2017), which doesn't use arrays of the twisting sequences. However, a more satisfactory solution is the aforementioned program $P 040201$, which implies the repeated generation of $U$ and $V$, whereas nsDeonYuliTwist $32 D$ doesn't provide this. Thus, we come to the inference that before performing a generation of the twisting planes, it is necessary to have tools for the trivial operations with the twisting sequences.

\section{Simple Twister Generator}

Class cDeonYuliSTwist32D, which is presented below, includes in its name a letter $S$ indicating the meaning of the word 'simple'. This class provides elementary operations with the twisting sequences and it does not use any arrays. The prototype of class cDeonYuliSTwist $32 D$ is class cDeonYuliTwist $32 D$. They are different in the issue that automatic setting of the congruential parameters $a$ and $c$ is excluded in class cDeonYuliSTwist32D. An example of using this class is presented in this section later, in the description of the program code P040301. namespace nsDeonYuliSTwist32D

\{ class cDeonYuliSTwist32D

$\{$ public uint $\mathrm{w}=16 \mathrm{U}$; public uint $\mathrm{N} 1=0 \mathrm{U}$; public uint $\mathrm{x} 0=1 \mathrm{U}$; uint $\mathrm{xB}=1 \mathrm{U}$; public uint $\mathrm{xG}=0 \mathrm{U}$; uint $\mathrm{xL}=0 \mathrm{U}, \mathrm{xR}=1 \mathrm{U}$; public uint $\mathrm{a}=5 \mathrm{U}$; public uint $\mathrm{c}=1 \mathrm{U}$; public uint maskW $=0 \mathrm{U}$; public uint maskU $=0 \mathrm{U}$; public uint maskT $=0 \mathrm{U}$;

public uint $\mathrm{nW}=0 \mathrm{U}$; // pair twister number in $\mathrm{w}$

// number bit length // max-number // sequence beginning // twister beginning

// created random number // pair numbers

$/ /$ congruential constant a

// congruential constant c // number mask // elder bit mask // twister bits public cDeonYuliSTwist32D()

$\{\mathrm{N} 1=0 x \mathrm{xFFFFFFF}>>(32$ - (int)w);//max-number \}

//----

public void StartCong(uint sxB)

$\{\mathrm{xB}=\mathrm{x} 0$; uint $\mathrm{sxBe}=\mathrm{sxB} \&$ maskW;

for (int $\mathrm{i}=0 ; \mathrm{i}<\mathrm{sxBe} ; \mathrm{i}++$ )

$\mathrm{xB}=(\mathrm{a} * \mathrm{xB}+\mathrm{c}) \&$ maskW;// shifted beginning $\mathrm{xR}=\mathrm{xB} ; \quad$ // for twister beginning \}

//----

public uint NextCong()

$\{\mathrm{xL}=\mathrm{xR} ; \quad$ // pair beginning $\mathrm{xR}=(\mathrm{a} * \mathrm{xL}+\mathrm{c}) \&$ maskW; $\quad$ // end of pair $\mathrm{xG}=\mathrm{xL}$;

$/ /$ created number \} return $\mathrm{xG}$;

$/ /--$

public void RepeatCong()

$\{\mathrm{xR}=\mathrm{xB} ; \quad$ // repeat track

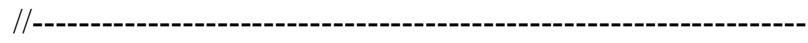

public void ShiftCong()

$\{\mathrm{xB}=(\mathrm{a} * \mathrm{xB}+\mathrm{c}) \&$ maskW; // shifted beginning \}

$/ /---$

public void StartTwist(uint snW)

$\{\mathrm{nW}=$ (uint)snW; $\quad / /$ bit shift size $\operatorname{maskT}=\operatorname{maskU} ; \quad / /$ elder 1 of twister mask for (int $\mathrm{m}=1 ; \mathrm{m}<\mathrm{nW} ; \mathrm{m}++$ )

maskT $\mid=\operatorname{maskU}>>\mathrm{m}$; $\quad$ // twister mask $\mathrm{xL}=\mathrm{xB} ; \quad / /$ for twister beginning \} $\mathrm{xR}=(\mathrm{a} * \mathrm{xL}+\mathrm{c}) \&$ maskW; // end of $\mathrm{xL}, \mathrm{xR}$ pair //----

public uint NextTwist()

\{ uint $\mathrm{g}=(\mathrm{xR} \&$ maskT $)>>($ int $)(\mathrm{w}-\mathrm{nW}) ; / /$ elder $\mathrm{xG}=((\mathrm{xL}<<($ int $) \mathrm{nW}) \&$ maskW $) \mid \mathrm{g} ; / /$ younger $\mathrm{xL}=\mathrm{xR} ; \quad$ // next pair beginning 


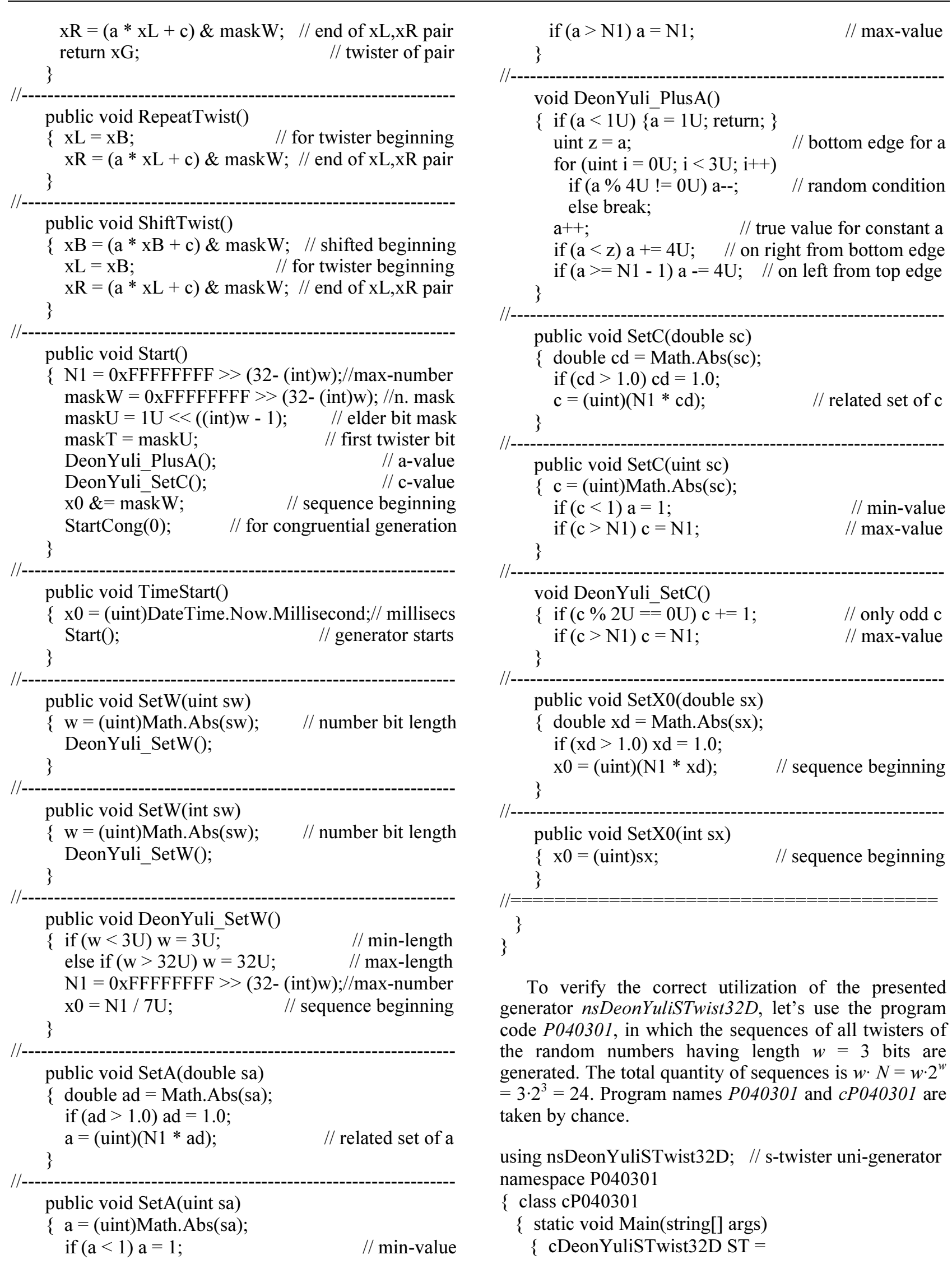




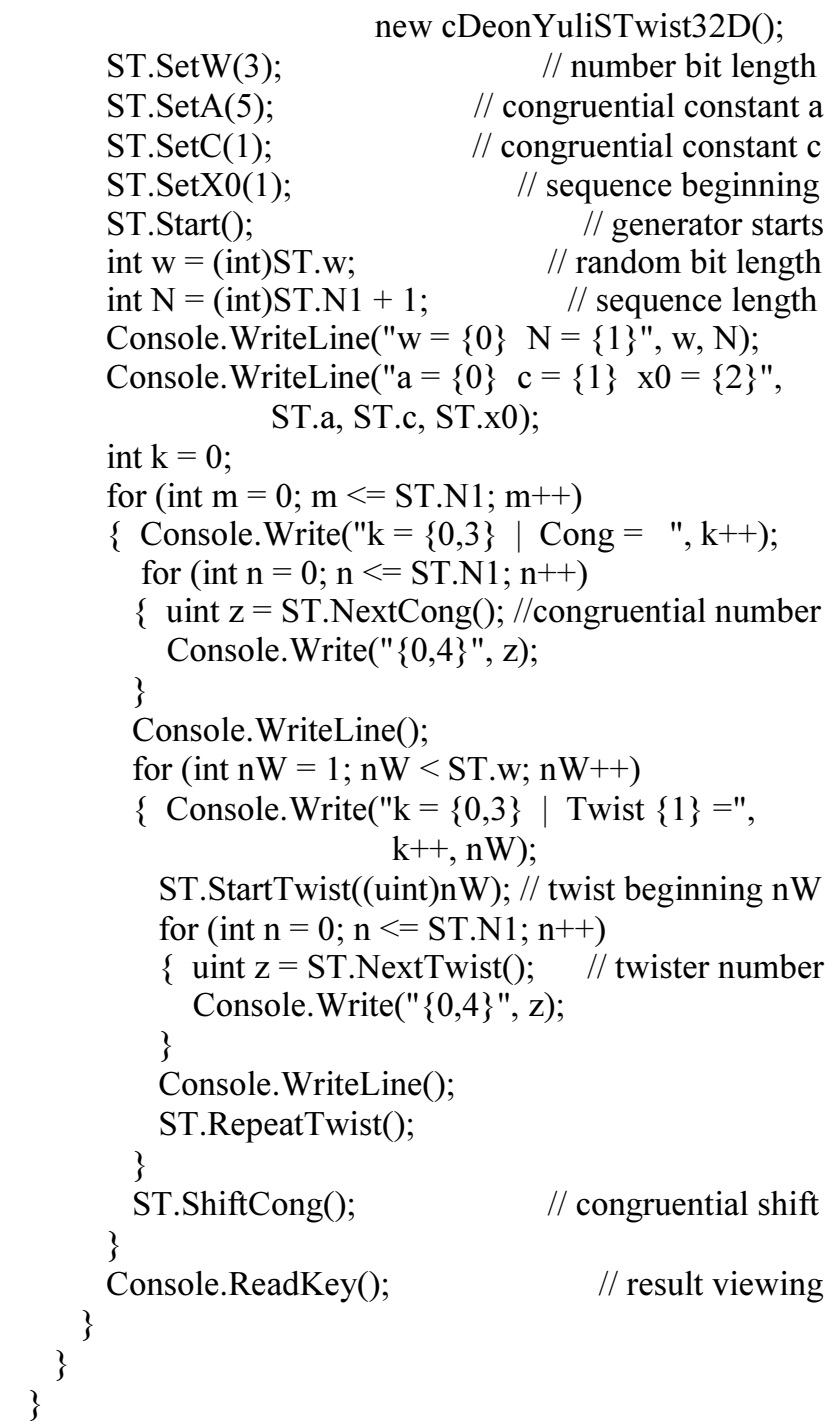

After the execution of P040301, the following listing appears on the monitor:

$\mathrm{W}=3 \mathrm{~N}=8$

$\mathrm{a}=5 \mathrm{c}=1 \mathrm{x} 0=1$

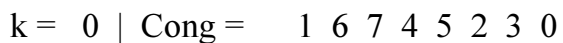

$\mathrm{k}=1 \mid$ Twist $1=\begin{array}{llllllll}3 & 5 & 7 & 1 & 2 & 4 & 6 & 0\end{array}$

$\mathrm{k}=2 \mid$ Twist $2=\begin{array}{lllllllll}7 & 3 & 6 & 2 & 5 & 1 & 4 & 0\end{array}$

$\mathrm{k}=3 \mid \mathrm{Cong}=\begin{array}{llllllll}6 & 7 & 4 & 5 & 2 & 3 & 0 & 1\end{array}$

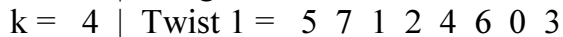

$\mathrm{k}=5 \mid$ Twist $2=\begin{array}{llllllll}3 & 6 & 2 & 5 & 1 & 4 & 0 & 7\end{array}$

$\mathrm{k}=6 \mid$ Cong $=\begin{array}{llllllll}7 & 4 & 5 & 2 & 3 & 0 & 1 & 6\end{array}$

$\mathrm{k}=7 \mid$ Twist $1=\begin{array}{llllllll}7 & 1 & 2 & 4 & 6 & 0 & 3 & 5\end{array}$

$\mathrm{k}=8 \mid$ Twist $2=\begin{array}{llllllll}6 & 2 & 5 & 1 & 4 & 0 & 7 & 3\end{array}$

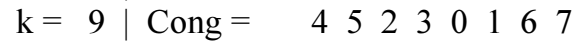

$\mathrm{k}=10 \mid$ Twist $1=\begin{array}{llllllll}1 & 2 & 4 & 6 & 0 & 3 & 5 & 7\end{array}$

$\mathrm{k}=11 \mid$ Twist $2=\begin{array}{llllllll}2 & 5 & 1 & 4 & 0 & 7 & 3 & 6\end{array}$

$\mathrm{k}=12 \mid$ Cong $=\begin{array}{llllllll}5 & 2 & 3 & 0 & 1 & 6 & 7 & 4\end{array}$

$\mathrm{k}=13 \mid$ Twist $1=\begin{array}{llllllll}2 & 4 & 6 & 0 & 3 & 5 & 7 & 1\end{array}$

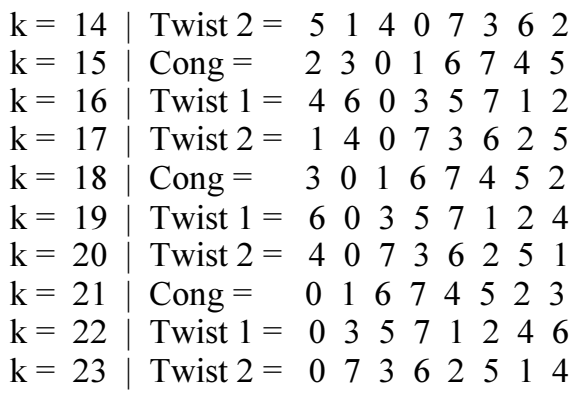

These congruential twisting sequences coincide with the result of tests of our previously developed twister generator $n s$ DeonYuliTwist28DA utilizing a technique of the congruential twisting array.

Running the program P040301 with other values of the bit length $w$ of the random numbers confirms a completeness of the generated sequences. This is sufficient to ensure the development of programs for generating the Descartes uniform twisting RPs.

\section{Twister Generator of Uniform Planes}

When constructing a complete generator of uniform twisting planes, let's use two uniform complete generators $G U$ and $G V$ of the aforementioned instrumental class cDeonYuliSTwist32D. It allows organizing the next class $n s$ DeonYuliPlaneTwist $32 D$ for the generation of all points on a grid of the twisting plane. By default, the initial track takes a diagonal of the grid points from the left-bottom position to the right-top one, although this may be changed by setting the independent beginnings for the internal generators $G U$ and $G V$. An example use of class cDeonYuliPlaneTwist $32 D$ is presented in this section later, located in the description of the program code $P 040302$.

using nsDeonYuliSTwist32D; // s-twister uni-generator namespace nsDeonYuliPlaneTwist32D

$\{$ class cDeonYuliPlaneTwist32D

$\{$ public uint $\mathrm{w}=16$; // uniform number bit length public uint $\mathrm{N} 1=0 ; \quad \quad / /$ max-number in track public uint $\mathrm{a}=5 \mathrm{U} ; \quad$ // congruential constant a public uint $\mathrm{c}=1 \mathrm{U} ; \quad$ // congruential constant $\mathrm{c}$ public uint $\mathrm{x} 0=1 \mathrm{U} ; \quad / /$ constant of track beginning public cDeonYuliSTwist32D GU; // generator 1 public cDeonYuliSTwist32D GV; // generator 2 public uint $\mathrm{nWU}=0 ; \quad / /$ twister shift number in GU public uint $\mathrm{nRU}=0 ; \quad / /$ ring shift number in $\mathrm{GU}$ public uint $\mathrm{nWV}=0 ; \quad / /$ twister shift number in $\mathrm{GV}$ public uint $\mathrm{nRV}=0 ; \quad / /$ ring shift number in $\mathrm{GV}$ public uint $\mathrm{nG}=0$; // elements in $\mathrm{GU}$ and $\mathrm{GV}$ tracks

public cDeonYuliPlaneTwist32D()
$\{\mathrm{GU}=$ new cDeonYuliSTwist32D(); // generator 1
$\mathrm{GV}=$ new cDeonYuliSTwist32D(); // generator 2
\}$\quad$
public void SetW(int sw)




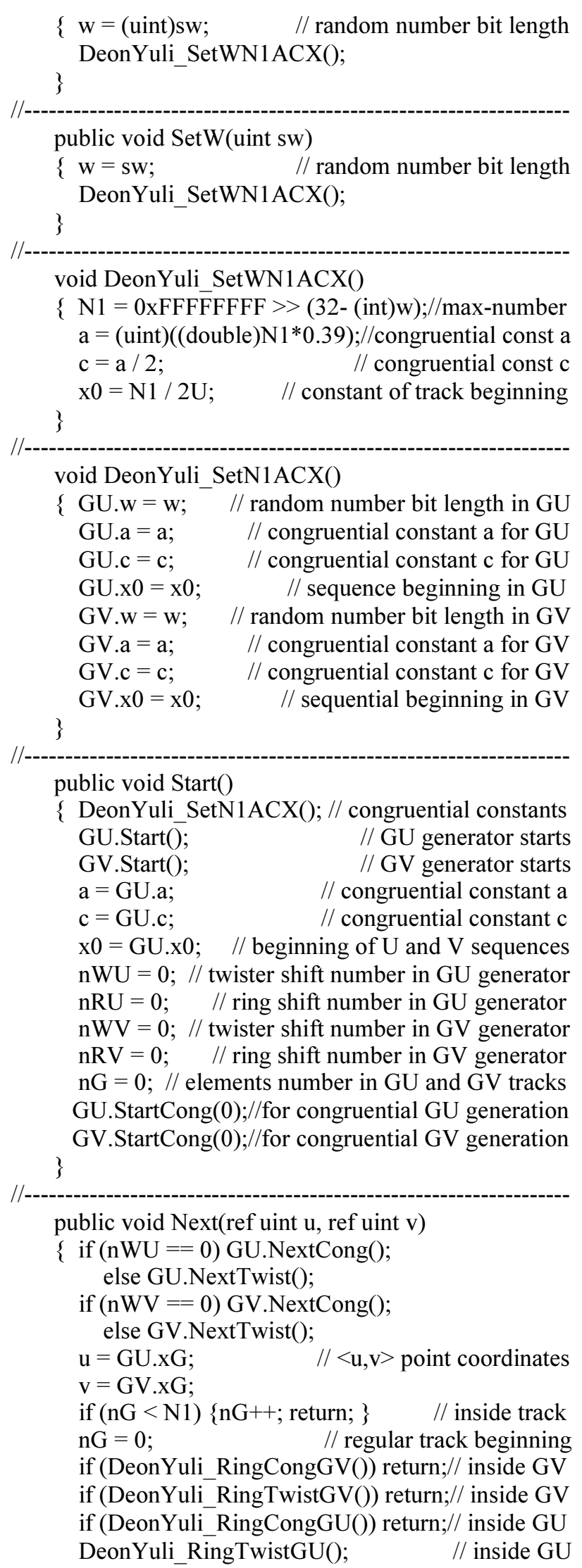

\}

bool DeonYuli_RingCongGV()

$\{$ if $(\mathrm{nWV} !=0)$ return false; // no congruential ring if (nWU = =0) GU.RepeatCong(); else GU.RepeatTwist();

if ( $\mathrm{nRV}<\mathrm{N1}$ )//congruent. ring opportunity in GV

\{ GV.ShiftCong(); // congruential shift in GV nRV++; // next ring number \} return true; // inside congruential track in GV
$\mathrm{nRV}=0$;

$\mathrm{nWV}=1$;

GV.StartTwist(nWV); return true;
// first ring in GV // first twister in GV // twister starts in GV // inside GV
\{ if $(\mathrm{nWU} !=0)$ return false; // no congruential ring if $(\mathrm{nRU}<\mathrm{N} 1) / /$ congruent. ring opportunity in GU \{ GU.ShiftCong(); // congruential shift in GU nRU++; // next ring number \} return true; // inside congruential track in GU $\mathrm{nRU}=0$ $\mathrm{nWU}=1$;

first ring in $\mathrm{GU}$ GUartTwist(nWU); // twister starts in GU return true; // inside GU

bool DeonYuli_RingTwistGU()

\{ if $(\mathrm{nWU}==\overline{0})$ return false; // no twister ring if $(\mathrm{nRU}<\mathrm{N} 1) \quad / /$ twister ring opportunity in GU \{ GU.ShiftTwist(); // twister shift in GU nRU++; 


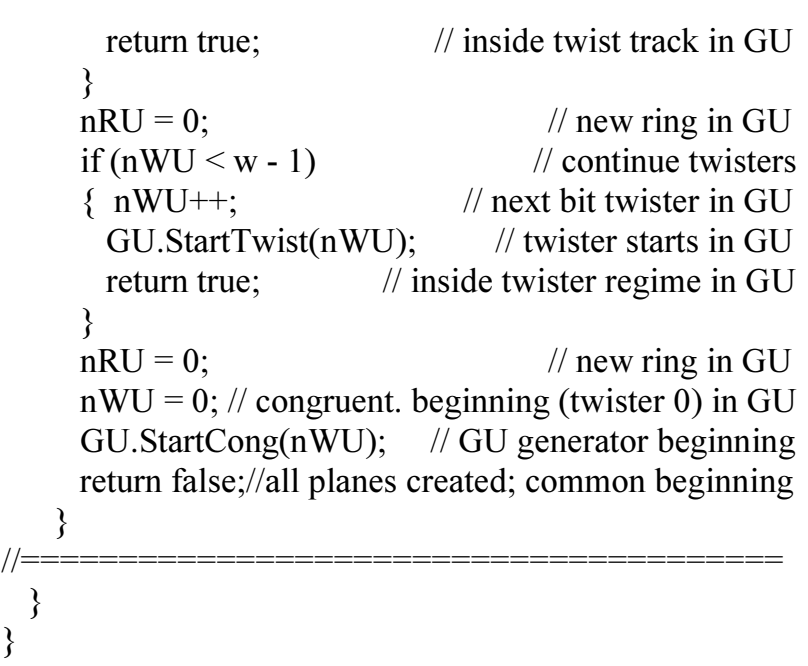

To test an operation of the presented generator $n s$ DeonYuliPlaneTwist $32 D$, let's use the program code P040302 shown below, in which the points of the initial twisting plane of the random numbers having length $w=$ 3 bits are generated. By default, the initial track of generation is a diagonal of the grid points from the leftbottom position to the right-top one. The uniformity of points on a plane would be confirmed by matrix $A$, in which the cell values are the counters of generation of the corresponding points $\langle u, v\rangle$ on RP. Program names $P 040302$ and $c P 040302$ are taken by chance.

using nsDeonYuliPlaneTwist32D;//twist-plane generator namespace $\mathrm{P} 040302$

\{ class cP040302

\{ static void Main(string[] args)

$\{$ cDeonYuliPlaneTwist32D TP $=$ new cDeonYuliPlaneTwist32D();

int $\mathrm{w}=3$;

// random number bit length

int $\mathrm{N}=1<<\mathrm{w}$;

// track length

TP.SetW(w);

TP.Start(); // generator starts

Console. WriteLine("w $=\{0\} \quad \mathrm{N}=\{1\}$ ", w, N);

Console.WriteLine("a $=\{0\} \quad \mathrm{c}=\{1\} \quad \mathrm{x} 0=\{2\} "$, TP.a, TP.c, TP.x0);

uint[] $\mathrm{u}=$ new uint[N]; // point $\mathrm{u}$-coords on track uint[] $\mathrm{v}=$ new uint[N]; // point $\mathrm{v}$-coords on track $\operatorname{int}[,] \mathrm{A}=$ new int[N, N]; $/ /$ result matrix for (int $\mathrm{i}=0 ; \mathrm{i}<\mathrm{N} ; \mathrm{i}++$ ) for (int $\mathrm{j}=0 ; \mathrm{j}<\mathrm{N} ; \mathrm{j}++$ ) $A[\mathrm{i}, \mathrm{j}]=0$;

uint $\mathrm{uu}=0 ; \quad$ // point job coordinates on plane uint $\mathrm{vv}=0$;

for (int $\mathrm{i}=0 ; \mathrm{i}<\mathrm{N} ; \mathrm{i}++$ ) // track values on plane

$\{$ Console. Write $(" \mathrm{i}=\{0,4\} \quad$ ", $\mathrm{i})$;

Console.Write(" nWU $=\{0,3\}$ ", TP.nWU);

Console.Write(" nRU $=\{0,3\} "$, TP.nRU);

Console.Write(" nWV $=\{0,3\} "$ ", TP.nWV);

Console.Write(" nRV $=\{0,3\}$ ", TP.nRV);

Console.WriteLine();

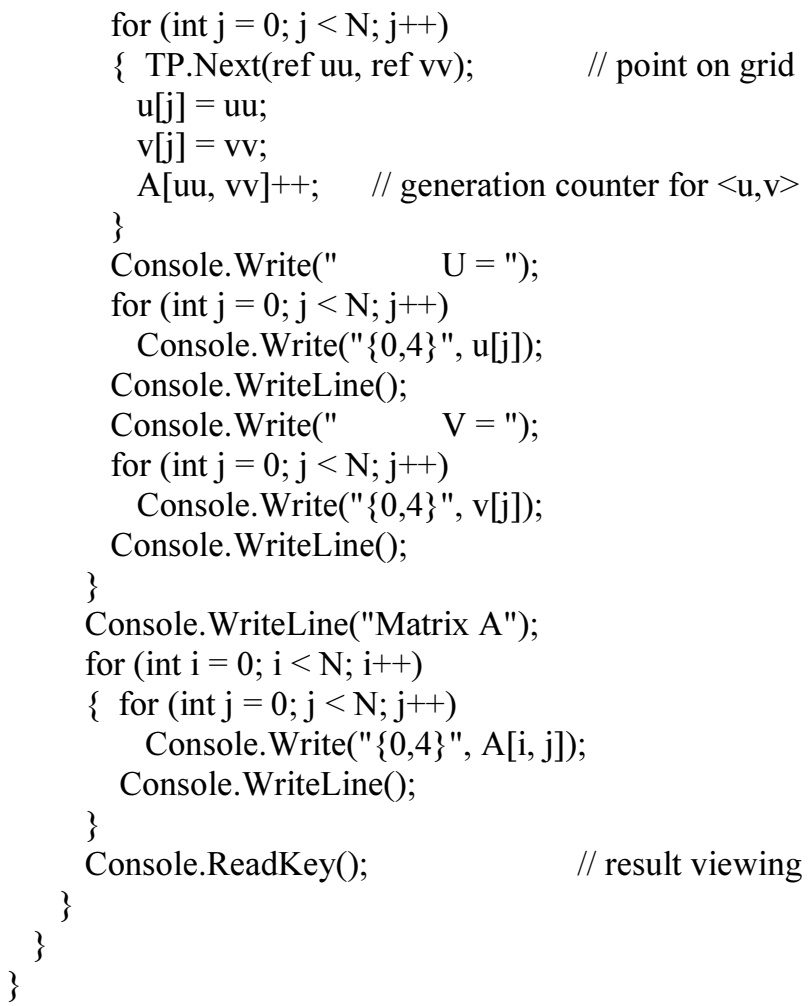

After the execution of P040302 code, the listing below appears. To reduce the listing size, we skipped some strings, which are indicated by a dashed line.

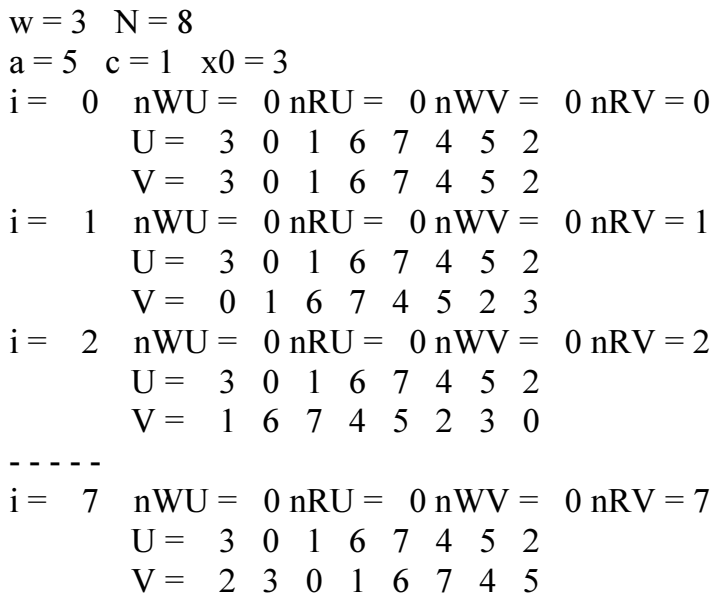

Matrix A

$\begin{array}{lllllllll}1 & 1 & 1 & 1 & 1 & 1 & 1 & 1\end{array}$

$\begin{array}{llllllll}1 & 1 & 1 & 1 & 1 & 1 & 1 & 1\end{array}$

$\begin{array}{llllllll}1 & 1 & 1 & 1 & 1 & 1 & 1 & 1\end{array}$

$\begin{array}{llllllll}1 & 1 & 1 & 1 & 1 & 1 & 1 & 1\end{array}$

$\begin{array}{llllllll}1 & 1 & 1 & 1 & 1 & 1 & 1 & 1\end{array}$

$\begin{array}{llllllll}1 & 1 & 1 & 1 & 1 & 1 & 1 & 1\end{array}$

$\begin{array}{llllllll}1 & 1 & 1 & 1 & 1 & 1 & 1 & 1\end{array}$

$\begin{array}{llllllll}1 & 1 & 1 & 1 & 1 & 1 & 1 & 1\end{array}$

In this listing, indicator $n W U$ shows the number of a 
twister on axis $U$ and indicator $n R U$ is pointed to the circular shift number on this axis $U$. The values $n W U=0$ and $n R U=0$ correspond to the congruential initial sequence on axis $U$. Similar values of indicators $n W V$ and $n R V$ show the circular shift of the initial sequence on axis $V$.

The listing of results for the singular matrix $A$ confirms that each random point $\langle u, v\rangle$ is created once. The same results are valid for other $w \leq 32$. This corresponds to a concept of the Descartes uniform RP. Thus, generator $n s$ DeonYuliPlaneTwist $32 D$ is ready for implementation.

\section{Discussion}

Twister generator nsDeonYuliPlaneTwist32D is capable creating a set of uniform twisting RPs for each pair of the congruential constants $a, c \in[\overline{1, N-1}]=\left[\overline{1,2^{w}-1}\right]$ in the sequences of random numbers having $w$ bit length. All the initial values $x_{0} \in\left[\overline{0,2^{w}-1}\right]$ are automatically presented in circles of the twisters (Deon and Menyaev, 2016b). However, the question is how many twisting planes could be obtained for each pair of parameters $a$ and $c$ ?

In the previous section, it has been determined that when creating the initial RP $U \times V$, two random sequences $U$ and $V$ can be taken, which are created by the corresponding twisting generators. It is known that twister 0 is the initial uniform congruential sequence. Let's denote it as $U_{0}$. Another sequence $V_{0}$ could be chosen arbitrarily but on the condition that it is also uniform. If the sizes or quantity of elements in the initial sequences are the same card $\left(U_{0}\right)=\operatorname{card}\left(V_{0}\right)$, then it can be stated that among all the possible tracks of the random uniform plane there has to be a track that creates points on the second diagonal (from the left-bottom position to the right-top one) of the corresponding square discrete grid. This is somewhat reminiscent of the idea of a central abstract element and specifically in our case this second diagonal track is the central track of the discrete grid. In generator nsDeonYuliPlaneTwist $32 D$ this is exactly what is done, shown at the beginning of the listing of results for the previous program P0040302.

$\begin{array}{lllllllll}U_{0}= & 3 & 0 & 1 & 6 & 7 & 4 & 5 & 2 \\ V_{0}= & 3 & 0 & 1 & 6 & 7 & 4 & 5 & 2\end{array}$

This is not the only solution because as the central track, one could take the main diagonal (from the left-top position to the right-bottom one) of a grid. But since the initial sequences $U$ and $V$ are random, the order of the vertex passage $\langle 3,3>,<0,0>,<1,1>,<6,6>,<7,7>,<4$, $4>,<5,5>,<2,2>$ is also random.

Now let's fix the sequence $U$ while the sequence $V$ is shifted to the left by the circular technique by one position.
$U_{0}=\begin{array}{llllllll}3 & 0 & 1 & 6 & 7 & 4 & 5 & 2\end{array}$

$V_{1}=\begin{array}{lllllllll}0 & 1 & 6 & 7 & 4 & 5 & 2 & 3\end{array}$

This combination of pairs gives the points of the first track $\langle 3,0\rangle,\langle 0,1>,\langle 1,6>,<6,7>,<7,4>,<4,5\rangle,<5$, $2>,<2,3>$ on RP. None of these points can appear on the reverse main diagonal of a grid, since sequences $U_{0}$ and $V_{1}$ are uniform. Their uniformity follows from the determined properties of the complete twisting sequences (Deon and Menyaev, 2016b). Shifting of sequence $V$, provided that sequence $U_{0}$ is fixed, could be continued and so the second track on a grid might be obtained.

$U_{0}=\begin{array}{lllllllll}3 & 0 & 1 & 6 & 7 & 4 & 5 & 2\end{array}$

$V_{2}=\begin{array}{lllllllll}1 & 6 & 7 & 4 & 5 & 2 & 3 & 0\end{array}$

The second track contains the points, which also cannot occur on track 1 and track 0 of the reverse diagonal and again that is because of the properties of the complete uniform twisting sequences. In this example, only 8 options to present sequence $V$ are possible since the $9^{\text {th }}$ shift repeats the initial variation $V_{8}=V_{0}$.

So, the shift operations for sequences comply with the corresponding varieties of the twisting sequences. In the presented example, the shifts of the congruential sequence, which comply with the congruential generation from the corresponding initial values, are considered. Thus, the congruential initial generation of sequences $U_{0}$ and $V_{0}$ initiates the creation of one RP using the complete sequence of shifts of one of the original sequences $V_{j}$ while another sequence $U_{0}$ is fixed. By analyzing the result of the previous program P040301, it's easy to see that among all sequences $V_{k \in[\overline{0,23}]}$ there are all 8 congruential shifts $V_{k=0}, V_{k=3}, V_{k}$ $=6, V_{k=9}, V_{k=12}, V_{k=15}, V_{k=18}, V_{k=21}$ in amount of $N=2^{w}$ $=2^{3}=8$ random numbers having length of 3 bits in the complete sequence. As a result, it turns out that tracks $<U_{0}, V_{j}>=<U_{0}, V_{K}>$ form the initial plane $L_{0}$.

Similar arguments apply to plane $L_{1}$. If we refer again to the result of the previous program P040301, this example shows that plane $L_{1}$ is created using the congruential twisting tracks $<U_{k}=0, V_{k \in[1,4}, 7,10,13,16,19$, ${ }_{22}>$. Plane $L_{2}$ is created by using shifts of the next twister $<U_{k=0}, V_{k \in[2,5,8,11,14,17,20,23]}>$. Next is plane $L_{3}$, but to create it we need to perform the congruential circular shift of sequence $U_{0}$ by one random step to the left, which leads to obtaining the new distribution of the random values $U_{k}=3$ along the axis $U$. Then, the next plane $L_{3}$, which can be obtained with the help of tracks $<U_{k=3}, V_{k \in[0,3,6,9,12,15,18,21]}>$.

A summary of all the points obtained leads us to the conclusion that number card $(L)$ of the complete set of RPs is defined by multiplication of two things. The first is $\operatorname{card}\left(U_{C T}\right)=w \cdot N=w \cdot 2^{w}$, which is a quantity of the different options of the congruential twisting forms of 
sequence $U$; the second is $\operatorname{card}\left(V_{T}\right)=w$, which is a quantity of the various twisters in sequence $V$ including the congruential twister 0 , i.e.,: $\operatorname{card}(L)=\operatorname{card}\left(U_{C T}\right)$. $\operatorname{card}\left(V_{T}\right)=w N \cdot w=w^{2} N$.

Below is the program code P040101, in which the uniformity of all the random twisting planes $L(w=3)$ is checked. Matrix $A$ is helpful for this task. Since in the uniform RPs each vertex is created once, after the full enumeration of all the RPs the quantity of generations of each vertex has to be equal to the amount of planes. In other words, the counters of cells of matrix $A$ have to have the same values and moreover, they have to be equal to the quantity of the generated RPs. To clarify, the program below uses the random numbers with a bit length $w=3$. The names P040401 and $c P 040401$ are selected by chance.

using nsDeonYuliPlaneTwist32D;//twist-plane generator namespace P040401

\{ class cP040401

\{ static void Main(string[] args)

$\{$ cDeonYuliPlaneTwist32D TP $=$ new cDeonYuliPlaneTwist32D();

int $\mathrm{W}=3 ; \quad \quad / /$ random number bit length int $\mathrm{N}=1<<\mathrm{w} ; \quad$ // track length TP.SetW(w);

TP.Start(); $/ /$ generator starts

Console.WriteLine("w $=\{0\} \quad \mathrm{N}=\{1\} ", \mathrm{w}, \mathrm{N})$;

Console. WriteLine("a $=\{0\} \quad \mathrm{c}=\{1\} \quad \mathrm{x} 0=\{2\} "$, TP.a, TP.c, TP.x0);

uint[] $\mathrm{u}=$ new uint[N]; // point $\mathrm{u}$-coords on track uint[] $\mathrm{v}=$ new uint[N]; // point $\mathrm{v}$-coords on track uint[,] $\mathrm{A}=$ new uint[N, N];

for (int $\mathrm{i}=0 ; \mathrm{i}<\mathrm{N} ; \mathrm{i}++$ ) for (int $\mathrm{j}=0 ; \mathrm{j}<\mathrm{N} ; \mathrm{j}++$ ) $A[\mathrm{i}, \mathrm{j}]=0$;

uint $\mathrm{uu}=0 ; \quad / /$ point job coordinates on plane uint $\mathrm{vv}=0$;

int plane $=0$;

int $\mathrm{k}=0$;

// plane number

while (true)

// track number

\{ uint $\mathrm{nWU}=$ TP.nWU, nRU = TP.nRU;

uint $n W V=$ TP.nRV, nRV = TP.nRV;

for (int $\mathrm{j}=0 ; \mathrm{j}<\mathrm{N} ; \mathrm{j}++$ )

\{ TP.Next(ref uu, ref vv); // point on grid

$$
\mathrm{u}[\mathrm{j}]=\mathrm{uu} \text {; }
$$$$
\mathrm{v}[\mathrm{j}]=\mathrm{vv}
$$$$
\text { \} }
$$$$
\mathrm{A}[\mathrm{uu}, \mathrm{vv}]++ \text {; }
$$

if $(\mathrm{k} \% \mathrm{~N}==0)$ plane $++; \quad / /$ plane number Console.WriteLine("plane $=\{0\}$ ", plane);

$$
\mathrm{k}++ \text {; }
$$

// next track

// if $(\mathrm{k}<569)$ continue;

Console. Write("k = $\{0,4\} \quad$ ", $\mathrm{k})$;

Console.Write("nWU = $\{0,3\}$ ", nWU);

Console.Write("nRU = $\{0,3\} \quad$ ", nRU);

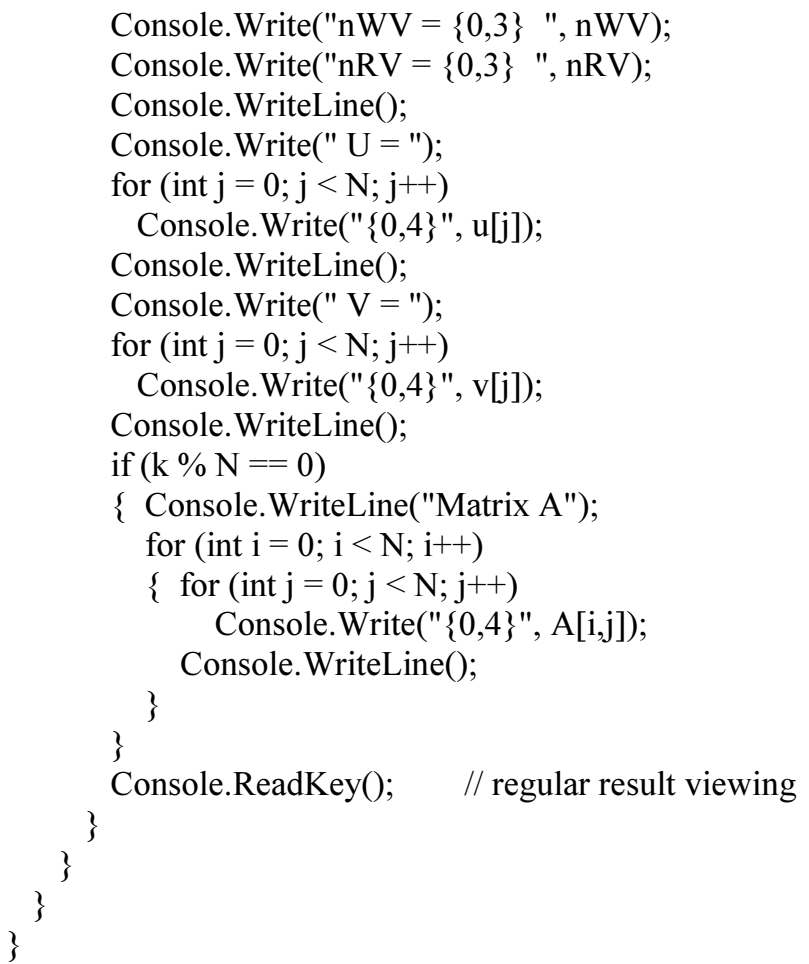

After executing the program P040401, the following listing below appears on the monitor. The skipped strings are indicated by a dashed line.

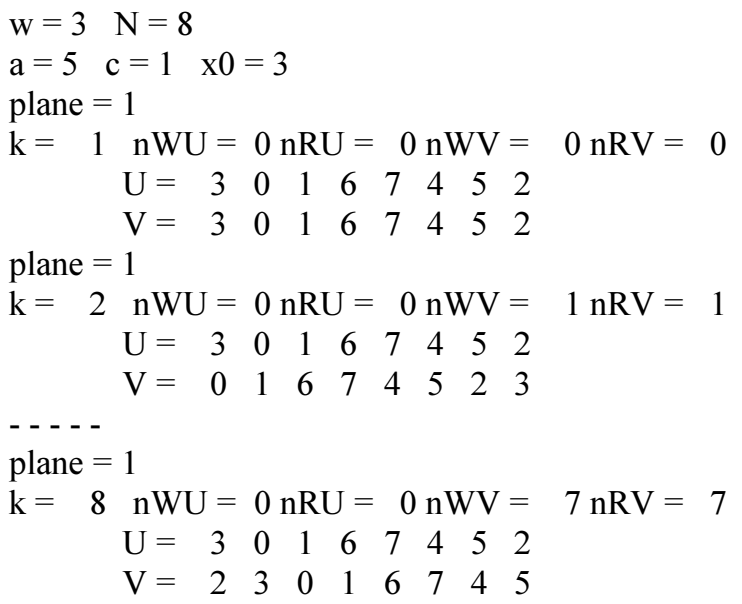




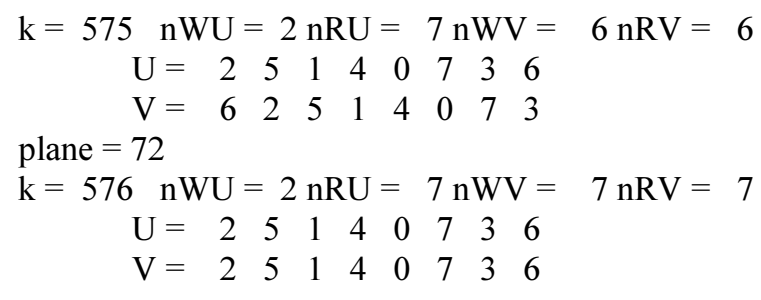

Matrix A

$\begin{array}{llllllll}72 & 72 & 72 & 72 & 72 & 72 & 72 & 72\end{array}$

$\begin{array}{llllllll}72 & 72 & 72 & 72 & 72 & 72 & 72 & 72\end{array}$

$\begin{array}{llllllll}72 & 72 & 72 & 72 & 72 & 72 & 72 & 72\end{array}$

$\begin{array}{llllllll}72 & 72 & 72 & 72 & 72 & 72 & 72 & 72\end{array}$

$\begin{array}{llllllll}72 & 72 & 72 & 72 & 72 & 72 & 72 & 72\end{array}$

$\begin{array}{llllllll}72 & 72 & 72 & 72 & 72 & 72 & 72 & 72\end{array}$

$\begin{array}{llllllll}72 & 72 & 72 & 72 & 72 & 72 & 72 & 72\end{array}$

$\begin{array}{llllllll}72 & 72 & 72 & 72 & 72 & 72 & 72 & 72\end{array}$

So, the values of the elements of matrix $A$ confirm the analytical calculations for $\operatorname{card}(L)=w^{2} N$ and for arbitrary $w \leq 32$. Generator nsDeonYuliPlaneTwist $32 D$ creates a complete set of uniform twisting RPs, which is considered as the primary task of this article.

\section{Conclusion}

Analysis of the sources indicates that algorithms of the generators of uniform planes do not take into account the potential of the sequences having absolutely uniform distribution. Techniques of those generating algorithms do not guarantee the absolute uniformity of the complete random planes. To overcome this limitation, we proposed here the generators of the complete uniform sequences, which include unique twisting techniques described in our previous works. However, their direct application for the described task is hampered by the required properties of Descartes uniform planes. To satisfy this requirement, a new class nsDeonYuliStwist32D was constructed and now with its help it is possible to create the dynamic objects of the simplest twisters without using congruential arrays. Applying secondary indexing technique allows for getting a generator of Descartes twisting random planes, which ensures the completeness and uniqueness of all the random variables on a grid of the Descartes plane. The performed tests confirm the absolute uniform distribution of the generated random values on a plane. In addition, a variety of the initial twisting sequences allows getting a set of the twisting planes for each pair of the congruential constants. In perspective, the obtained results can be used in a large number of applied tasks, which use the spatial plane distributions.

\section{Acknowledgment}

The authors are thankful to Matthew Vandenberg, Jacqueline Nolan, Julia Alex Watts and Walter
Harrington (University of Arkansas for Medical Sciences, Little Rock, AR, USA) for the proofreading.

\section{Author's Contributions}

Both authors equally contributed to this work.

\section{Funding Information}

The authors have no support or funding to report.

\section{Ethics}

This article is original and contains unpublished material. The corresponding author confirms that all of the other authors have read and approved the manuscript. No ethical issues were involved and the authors have no conflict of interest to disclose.

\section{References}

Alani, M.M., 2010. Testing randomness in ciphertext of block-ciphers using diehard Tests. Int. J. Comput. Sci. Netw. Secur., 10: 53-57.

Bekkerman, R., M. Sahami and E. Learned-Miller, 2006. Combinatorial Markov random fields. Proceedings of the 17th European Conference on Machine Learning, (CML' 06:), pp: 30-41. DOI: $10.1007 / 118718428$

Berger, E.D. and B.G. Zorn, 2006. DieHard: Probabilistic memory safety for unsafe languages. Proceedings of the 27th ACM SIGPLAN Conference on Programming Language Design and Implementation, (LDI' 06), pp:158-168. DOI: $10.1145 / 1133255.1134000$

Dachian, S. and B.S. Nahapetia, 2009. On gibbsianness of random fields. Markov Process. Relat., 15: 81-104.

Deon, A.F. and Y.A. Menyaev, 2016a. The complete set simulation of stochastic sequences without repeated and skipped elements. J. Univers. Comput. Sci., 22: 1023-1047. DOI: 10.3217/jucs-022-08-1023

Deon, A.F. and Y.A. Menyaev, 2016b. Parametrical tuning of twisting generators. J. Comput. Sci., 12: 363-378. DOI: $10.3844 /$ jcssp.2016.363.378

Deon, A.F. and Y.A. Menyaev, 2017. Twister generator of arbitrary uniform sequences. J. Univers. Comput. Sci., 23: 353-384.

Feller, W., 2008. An Introduction to Probability Theory and Its Applications. 3rd Edn., WSE Press, ISBN-10: 8126518057, pp: 509.

Gnedenko, B., 1998. Theory of Probability. 6th Edn., CRC Press, ISBN-10: 9056995855, pp: 520.

Kim, J. and R. Zabih., 2002. Factorial Markov random fields. Proceedings of the European Conference on Computer Vision, (CCV' 02), pp: 321-334. DOI: $10.1007 / 3-540-47977-5$ 21 
Koonce, N.A., M.A. Juratli, C. Cai, M. Sarimollaoglu and Y.A. Menyaev et al., 2017. Real-time monitoring of Circulating Tumor Cell (CTC) release after nanodrug or tumor radiotherapy using in vivo flow cytometry. Biochem. Biophys. Res. Commun., 492: 507-512. DOI: $10.1016 /$ j.bbrc.2017.08.053

Kumar, S. and M. Hebert, 2003. Discriminative random fields: A discriminative framework for contextual interaction in Classification. Proceedings of the 9th IEEE International Conference on Computer Vision, (CCV' 03), pp: 1150-1157. DOI: 10.1109/ICCV.2003.1238478

Matsumoto, M. and T. Nishimura, 1998. Mersenne twister: A 623-dimensionally equidistributed uniform pseudorandom number generator. ACM TOMACS, 8: 3-30. DOI: 10.1145/272991.272995

Matsumoto, M., M. Saito, H. Haramoto and T. Nishimura, 2006. Pseudorandom number generation: Impossibility and compromise. J. Univers. Comput. Sci., 12: 672-690. DOI: 10.3217/jucs-012-06-0672

Matsumoto, M., I. Wada, A. Kuramoto and H. Ashihara, 2007. Common defects in initialization of pseudorandom number generators. ACM TOMACS. DOI: $10.1145 / 1276927.1276928$

Menyaev, Y.A. and V.P. Zharov, 2005. Phototherapeutic technologies for oncology. Proceedings of SPIE, 5973:271-278. DOI: 10.1117/12.640217

Menyaev, Y.A. and V.P. Zharov, 2006a. Experience in development of therapeutic photomatrix equipment. Biomed. Eng., 40: 57-63. DOI: $10.1007 / \mathrm{s} 10527-006-0042-6$

Menyaev, Y.A. and V.P. Zharov, 2006b. Experience in the use of therapeutic photomatrix equipment. Biomed. Eng., 40: 144-147. DOI: 10.1007/s10527-006-0064-0

Menyaev, Y.A., D.A. Nedosekin, M. Sarimollaoglu, M.A. Juratli and E.I. Galanzha et al., 2013. Optical clearing in photoacoustic flowcytometry. Biomed. Opt. Express, 4: 3030-41. DOI: 10.1364/BOE.4.003030

Menyaev, Y.A., K.A. Carey, D.A. Nedosekin, M. Sarimollaoglu and E.I. Galanzha et al., 2016. Preclinical photoacoustic models: Application for ultrasensitive single cell malaria diagnosis in large vein and artery. Biomed. Opt. Express, 7: 3643-58. DOI: 10.1364/BOE.7.003643

Newman, M.E.J. and G.T. Barkema, 1996. Monte carlo study of the random-field ising model. Phys. Rev. E., 53: 393-404. DOI: 10.1103/PhysRevE.53.393
Novark, G. and E.D. Berger, 2010. DieHarder: Securing the heap. Proceedings of the 17th ACM Conference on Computer and Communications Security, (CCS'10), pp: 573-584. DOI: 10.1145/1866307.1866371

Qi, Y., M. Szummer and T.P. Minka, 2004. Bayesian conditional random fields. Proceedings of the 10th International Workshop on Artificial Intelligence and Statistics, (ATS'05), pp: 1-8.

Quattoni, A., M. Collins and T. Darrell, 2004. Conditional random fields for object recognition. Proceedings of the Advances in Neural Information Processing Systems, (NIPS' 04), pp: 1-8.

Rimstad, K. and H. Omre, 2014. Skew-gaussian random fields. Spat. Stat., 10: 43-62. DOI: $10.1016 /$ j.spasta.2014.08.001

Saito, M. and M. Matsumoto, 2008. SIMD-Oriented Fast Mersenne Twister: A 128-Bit Pseudorandom Number Generator. In: Monte Carlo and Quasi-Monte Carlo Methods, Keller, A., S. Heinrich and H. Niederreiter, (Eds.), Springer Science and Business Media, Berlin, ISBN-10: 3642041078, pp: 672.

Sarawagi, S. and W.W. Cohen, 2004. Semi-Markov conditional random fields for information extraction. Proceedings of the Advances in Neural Information Processing Systems, (NIPS' 04), pp: 9-18.

Sha, F. and F. Pereira, 2003. Shallow parsing with conditional random fields. Proceedings of the Conference of the North American Chapter of the Association for Computational Linguistics on Human Language Technology, (ACL'03), pp:134-141. DOI: $10.3115 / 1073445.1073473$

Spanos, P.D. and B.A. Zeldin, 1998. Monte carlo treatment of random fields: A broad perspective. Applid Mech. Rev., 51: 219-237. DOI: 10.1115/1.3098999

Sung, Y.H. and D. Jurafsky, 2009. Hidden conditional random fields for phone recognition. Proceedings of the IEEE Workshop on Automatic Speech Recognition and Understanding, (SRU' 09), pp: 107-112. DOI: 10.1109/ASRU.2009.5373329

Sutton, C. and A. McCallum, 2012. An introduction to conditional random fields. Found. Trends Mach. Learn., 4: 267-373. DOI: 10.1561/2200000013

Xiao, Y., 2010. Uniform modulus of continuity of random fields. Monatsh. Math., 159: 163-184. DOI: $10.1007 / \mathrm{s} 00605-009-0133-\mathrm{z}$ 